\title{
$\checkmark$ Research Square

\section{Attitudes, Perceptions of Risk, and Behavior Change of Students During Pandemics: A Systematic Review}

\section{Simran Bakshi}

University of Western Ontario

Aghna Wasim

University of Toronto https://orcid.org/0000-0002-6885-9503

Judy Truong

MaRS Discovery District

Umair Majid ( Umair.majid@mail.utoronto.ca )

University of Toronto https://orcid.org/0000-0002-4581-7714

\section{Systematic Review}

Keywords: COVID-19, pandemic, education, risk perception, behaviour change, students

Posted Date: January 4th, 2021

DOI: https://doi.org/10.21203/rs.3.rs-139671/v1

License: (c) (i) This work is licensed under a Creative Commons Attribution 4.0 International License. Read Full License 


\section{Abstract}

Students in higher education institutions such as universities are at a unique risk for COVID-19 because learning takes place in a manner that warrants frequent social interaction. This systematic review of 18 studies examined the awareness, risk perception, and health behaviors among students in higher education institutions across 15 high- and low-resource countries. We found that accurate knowledge of the disease varied across different countries. We also found that students were more likely to use informal networks and social media to obtain quick and easy-to-understand information about the infection. However, a "casual" or low risk attitude was prevalent from the start of each pandemic of outbreak. This casual attitude circumscribed the adoption of prevention behaviors. We conclude our paper by using the Information-Motivation-Behavioral Model to recommend how to maximize the impact of the design and delivery of public health interventions in the student population.

\section{Background}

As of December $28^{\text {th }}$, there have been more than 80 million cases of the coronavirus disease 2019 (COVID-19) reported worldwide (Johns Hopkins University's Center for Systems Science and Engineering). In response, governments all around the world have launched public health emergency responses to combat the spread of the virus and have implemented physical distancing, community lockdown, quarantine protocols, public health education, and school and workplace closures. The coronavirus pandemic has severely impacted workers in the education field and has forced students, teachers and other administrators to an unprecedented state. Teachers have had to field tough questions from parents and quickly transition to remote learning, and these changes alone have greatly impacted student learning globally. In particular, students in higher education institutions such as universities are at a unique risk. A vast amount of literature has found a notable correlation where pandemic knowledge increases risk perception, which may potentially influence behavior change (Majid et al., 2020b). University students make up a cohort of individuals who are just beginning to explore their independence, and as a result, their lack of life experience will greatly impact their perception of disease risk and adoption of appropriate health hygiene behaviours (Majid et al., 2020a). A study conducted in Bangladesh in the midst of the COVID-19 pandemic demonstrated that although $54.1 \%$ of students felt that they were highly exposed to COVID-19, $64.9 \%$ of students displayed a low level of knowledge and more than one-third of students were not interested in staying home and avoiding crowds (Wadood et al., 2020). This finding indicates that high risk perception of disease may not lead to the adoption of individual hygiene and physical distancing behaviors due to a lack of knowledge and awareness about COVID-19. Similarly, a H1N1 study conducted in Korea showed that during the peak of the pandemic period, only $30.3 \%$ of participants reported increasing their hand washing frequency (Park et al., 2010). In terms of risk perception, $64.0 \%$ of participants in the study by Park et al. (2010) indicated that an H1N1 influenza infection would have "no impact" or "only a minor impact" on their daily life. For example, a study conducted in Syria found many students did not make behavioral changes during the COVID-19 
pandemic where only $7.3 \%$ of students indicated wearing a mask when going out and $27.8 \%$ of students did not abide by physical distancing recommendations (Alhamid, 2020).

University students - as well as workers in the field of education - are a community that require unique public health interventions that promote the adoption of individual hygiene behaviors. Individuals in educational institutions are at a higher risk of contracting disease because traditionally, learning takes place in a manner that facilitates high social contact. However, there is a need for clarity on how these groups respond to pandemics, epidemics, and outbreaks, which may aid in optimizing existing interventions in higher education institutions to improve the reach and uptake of physical distancing and other individual hygiene behaviors. This systematic review will examine the awareness, risk perception, and health behaviors among students in higher education institutions during three major pandemics and outbreaks in the 21st century: Ebola Virus Disease, Influenza A/H1N1, and MERS.

\section{Methods}

\section{Approach}

We conducted a systematic review to examine the awareness, risk perception, and health behaviors among university students and other workers in higher education institutions during three major pandemics and global outbreaks that occurred in the 21st century: Ebola, Influenza A/H1N1 and MERS. We included qualitative, quantitative, and mixed-methods studies that analyzed awareness, risk perception and hygiene behaviours during pandemics and global outbreaks in higher educational institutions such as universities and colleges.

\section{Searching}

We recently published a scoping review that examined the response of various individuals and communities of five major pandemics, epidemics and outbreaks in the 21st century: Ebola, Influenza A/H1N1, MERS, SARS, and COVID-19 (Majid et al., 2020b). We decided to remove articles that looked specifically at university students and other workers in higher education institutions from that review because they constitute a rather unique group that may differ from the general public. We used the articles retrieved in that review for this systematic analysis. Four databases: PsychINFO, Embase, Global Health and MEDLINE were searched on March 7, 2020 as a part of the original scoping review. Our search strategy is available in a supplementary file.

\section{Screening}

A pair of researchers used Covidence to conduct title and abstract screening, and full-text screening. We were most interested in qualitative, quantitative, as well as mixed-methods studies analyzing how students in higher education institutions responded to Ebola, H1N1, and MERS. We included articles describing social media analyses. We excluded theses, dissertations, abstracts as well as published papers that did not have any empirical primary data. 


\section{Data Extraction}

We extracted study and methodological characteristics using a data extraction form, including the following information: author, year of publication, title, research objectives, country, type of pandemic, epidemic or outbreak (Ebola, Influenza A/H1N1, or MERS), topic/setting (community knowledge and attitudes, risk perception, public trust, social media, and community interventions), methodology (crosssectional, qualitative or mixed-methods design), data collection method (semi-structured interviews, selfadministered questionnaire, or focus groups), and number and type of participants.

\section{Data Analysis}

We conducted qualitative analysis of findings across the following stages: pilot coding, initial coding, and focused coding. In the pilot coding stage, we identified common themes in the abstracts and used them to develop a preliminary coding schema. We used the schema to create two coding templates: 1) knowledge and awareness and 2) health behaviours to code the results and discussion sections of included studies (Aronson, 1995). The coding process was completed with consideration for how the authors presented their findings. We extracted relevant data from the included studies and used it to populate our templates in the focused coding stage. Using the constant comparative method, we then conducted a comparison of codes across various studies, contexts, topics and countries (Boejie, 2002). Following the completion of coding, the lead author drafted narrative summaries for every theme, which they revised in light of feedback provided by other researchers. The lead author then integrated all narrative summaries in order to provide a clear and coherent interpretation of the results.

\section{Quality_Appraisal}

We appraised observational studies using the STROBE checklist retrieved from the Equator Network (Von Elm et al., 2007). We used the cohort and cross-sectional versions of STROBE as appropriate. Each study was appraised as a yes, no, or not applicable in accordance with the quality criteria. The appraisal findings are reported narratively in a supplemental file.

\section{Results}

\section{Knowledge and Attitudes}

A majority of studies indicated that students possessed moderate to high levels of overall knowledge regarding $\mathrm{H} 1 \mathrm{~N} 1$ that ranged from $75 \%$ in Mexico (Wilson and Huttlinger, 2010) to $99.6 \%$ in Australia (Van et al., 2010). However, this was not always the case; for example, one study conducted in the United Kingdom reported low levels of H1N1-related knowledge with $37.4 \%$ of students failing to identify any causes of the infection (Williams et al., 2012). Similar to H1N1, knowledge about MERS was variable, however it was considerably lower, ranging from $43.2 \%$ in Saudi Arabia (Al-Mohaissen, 2016) to 53.5\% in South Korea (Yang and Cho, 2017). In contrast, 93.2\% of students in one study from Iran expressed 
awareness of EVD and was thus, indicative of high levels of overall knowledge about the disease (Holakouie-Naieni et al., 2015).

Knowledge regarding the means of transmission of various pandemics was generally high. A total of six studies reported that a majority of participants were knowledgeable about the modes of transmission (Akan et al., 2010; Al-Mohaissen, 2016; Hasan et al., 2018; Holakouie-Naieni et al., 2015; Hussain et al., 2012; Seale et al., 2012). Human-to-human transmission of H1N1 was identified by a range of $52 \%$ of students in Pakistan (Hussain et al., 2012) to $84.4 \%$ in Turkey (Akan et al., 2010). In Iran, 56.2\% of undergraduate and $88.9 \%$ of graduate students were aware of Ebola transmission (Holakouie-Naieni et al., 2015). $60.5 \%$ of students from Saudi Arabia could identify different routes of MERS transmission including animal to human transmission, as well as transmission from infected family members, patients, and health care professionals (Al-Mohaissen, 2016).

Multiple studies noted high levels of knowledge about the signs and symptoms of H1N1, MERS, and EVD (Al-Mohaissen, 2016; Hasan et al., 2018; Holakouie-Naieni et al., 2015; Hussain et al., 2012). For example, a study conducted in Iran suggested that $77.7 \%$ of the students were aware of the signs and symptoms of EVD (Holakouie-Naieni et al., 2015). Moreover, fever, cough, difficulty in respiration and occasional diarrhea were recognized as symptoms of MERS by $78.9 \%$ of students in Saudi Arabia (Al-Mohaissen, 2016). Knowledge regarding the symptoms of $\mathrm{H} 1 \mathrm{~N} 1$ was more variable; while cough was identified as a common symptom of H1N1 by $72 \%$ (Hasan et al., 2018) to 83\% (Hussain et al., 2012) of students in Pakistan, diarrhea and vomiting were identified by only $15.5 \%$ and $32.2 \%$ of students, respectively (Hasan et al., 2018).

We also identified several gaps in knowledge among students, pertaining to the nature of pandemics, transmission modes, antiviral drugs and high-risk populations (Akan et al., 2010; Hasan et al., 2018; Holakouie-Naieni et al., 2015; Seale et al., 2012; Serino et al., 2011). For example, university students from Australia were unable to accurately define what 'pandemic influenza' meant despite being knowledgeable about its transmission (Seale et al., 2012). Furthermore, a study conducted in Turkey indicated a lack of knowledge about antiviral drugs and antiviral treatment in nearly one-third of the students (Akan et al., 2010). Another study reported that $37.1 \%$ of students from Iran had no knowledge regarding populations that were at the greatest risk of EVD (Holakouie-Naieni et al., 2015). Similarly, 45\% of students in one study conducted in Pakistan were unaware that touching an infected person was a mode of H1N1 transmission (Hasan et al., 2018).

\section{Information Sources}

Students derived information about the pandemic from various sources such as social media, newspapers, university resources and health care workers (Akan et al., 2010; Al-Mohaissen, 2016; Mitchell et al., 2011; Seale et al., 2011; Serino et al., 2011; Wilson and Huttlinger, 2010). Sources of information about pandemics and outbreaks such as health care workers, social media, community leaders, and the government each play an important role in disseminating information related to the infection and disease. A study conducted in the United States found $96.8 \%$ of students received information from the 
university about the H1N1 pandemic via email, $82.6 \%$ via telephone messages, and $80.7 \%$ via text messages (Mitchell et al., 2011). For example, in one study conducted in Australia, students expressed doubt towards the impact that the disease had on communities (Seale et al., 2012). The university sent out an email to staff and students educating them about the disease, but only $53.6 \%$ of students who received it found that it was useful (Seale et al., 2012). Another study found students seldom accessed reputable information sources such as medical search engines, the Centers for Disease Control and Prevention, and health care workers (Akan et al., 2010). For example, only $8 \%$ of students in Turkey accessed health care workers as their primary source of information (Akan et al., 2010). A study conducted in Spain found students associated the emergence of EVD with government mismanagement and misinformation from mass media (Mondragon et al., 2017).

Social media was the most widely used source of information amongst students. Three studies from Italy, Turkey, and the United States reported that students used social media as their primary source of information (Akan et al., 2010; Serino et al., 2011; Wilson and Huttlinger, 2010). In Turkey, 72.1\% of students reported using mass media as an information source, and $19.9 \%$ of students relied on the internet (Akan et al., 2010). In New Mexico University, a rural based community, students relied on electronic social networking through talking to individuals online or using electronic media (Wilson and Huttlinger, 2010). In Italy, 81\% of students were informed about H1N1 through mass media, and often multiple information sources were also used (Serino et al., 2011). These findings emphasize the significance of social media use during pandemics which played a vital role in information dissemination to students. Social media is not only a tool for dissemination, distribution and seeking of health information, but it may also act as an outlet for the students' emotions and sharing of personal experiences and opinions, which makes controlling the acquisition of information more difficult (Yang and Cho, 2017). These emotions may lead to expressions of skepticism about scientific topics and participation in decision-making within social media where misinformation may be widespread (Yang and Cho, 2017).

\section{Risk Perception}

\section{Risk Perception and Attitude}

Eight studies identified a "casual attitude" or low risk perception among students in response to pandemic infection or disease (Akan et al., 2010; Mitchell et al., 2011; Park et al., 2010; Seale et al., 2012; Serino et al., 2011; Van et al., 2010; Williams et al., 2012; Wilson and Huttlinger, 2010). A study conducted in Australia found that although $60.4 \%$ of university students believed that the H1N1 pandemic was serious, $40.4 \%$ reported that they were "not anxious" (Van et al., 2010). Students in this study believed they were not susceptible to H1N1, despite being the age group with the higher number of cases (Van et al., 2010). In a separate study conducted in Australia, students believed they were capable of fighting off any illness because they were young and led a healthy lifestyle (Seale et al., 2012). Similar sentiments were also found in students residing in the United States (Mitchell et al., 2011; Wilson and Huttlinger, 2010). For example, a study conducted in the United States found that $60 \%$ of students did not perceive 
dormitory residences to be a source of infection despite there being evidence that shared bathrooms and sleeping quarters were high risk areas for disease spread (Wilson and Huttlinger, 2010).

\section{Influence of Mass Media on Risk Perception}

The influence of mass media on student perception of the infection varied throughout the pandemic. For example, a study conducted in Australia found that the resurgence of H1N1 media coverage caused a significant increase in anxiety, perceived susceptibility, and seriousness of disease spread (Van et al., 2010). However, media coverage of the infection declined in accordance with a decrease in laboratory confirmed cases of H1N1 in Australia (Van et al., 2010). Similarly, another study conducted in Pakistan reported $32 \%$ of medical students believed themselves to be at a risk of contracting H1N1 (Hussain et al., 2012). This finding reflected the relationship between low-risk perception due to the low number of laboratory-confirmed outbreaks reported in the newspaper and media (Hussain et al., 2012). Furthermore, one study conducted in South Korea reported a positive correlation between trust in the media, risk perception and overreaction (Yang and Cho, 2017). The study supported the claim that the mass media may increase perceptions of disease risks due to the frequent reporting of community spread of disease (Yang and Cho, 2017).

\section{Health Behaviors}

Studies reported that a significant portion of students did not wear masks. Two studies found that the use of a face mask was met with the most resistance among undergraduate and graduate students (Hasan et al., 2018; Van et al., 2010). University students from Australia dismissed the use of masks as they were considered uncomfortable, inconvenient, and unnecessary (Seale et al., 2012). Furthermore, students believed that wearing a mask would contribute to embarrassment and social stigma (Seale et al., 2012). The percentage of students who did not wear face masks in crowded areas ranged from $16.8 \%$ in Australia (Van et al., 2010) to 84.5\% in Pakistan (Hasan et al., 2018). Interestingly, the majority of the students agreed that the use of a facemask was an effective preventative measure, but only a $11.5 \%$ wore one when having fever, cough or a runny nose (Hasan et al., 2018).

Students in eight studies reported regular hand washing and sanitizing to prevent the spread of infection (Akan et al., 2010; Hasan et al., 2018; Mitchell et al., 2011; Park et al., 2010; Seale et al., 2012; Serino et al., 2011; Van et al., 2010; Zottarelli et al., 2012). The percentage of students who practiced regular handwashing ranged from $20.8 \%$ in Australia (Van et al., 2010) to $96 \%$ in the United States (Mitchell et al., 2011). Students in two studies conducted in South Korea and Italy reported an increase in handwashing frequency during the peak of the H1N1 influenza pandemic (Park et al., 2010; Serino et al., 2011). The proportion of students who reported an increase in this behavior ranged from 30.3\% (Park et al., 2010) to $38 \%$ (Serino et al., 2011). A study conducted in Australia found students perceived hand washing as the most feasible and acceptable preventive measure compared to physical distancing and mask use (Seale et al., 2012). 
Students in four studies reported avoiding close contact with their family and friends (Akan et al., 2010; Hasan et al., 2018; Mitchell et al., 2011; Van et al., 2010). The proportion of university students who reported this behavior ranged from 5\% in Turkey (Akan et al., 2010) to 80.5\% in Pakistan (Hasan et al., 2018). Furthermore, university students in two studies reported covering their mouth and nose while coughing or sneezing (Hasan et al., 2018; Mitchell et al., 2011). The proportion of students who reported this behaviour ranged from $77.7 \%$ in Pakistan (Hasan et al., 2018) to $80.6 \%$ in the United States (Mitchell et al., 2011). University students from three studies avoided crowded places (Hasan et al., 2018; Serino et al., 2011; Van et al., 2010). A study conducted in Australia reported approximately half of university student participants were willing to avoid large class sizes and resort to online learning instead (Van et al., 2010).

\section{The Relationship between Risk Perception and Health Behavior}

Students' low perception of infection risk plays a vital role in encouraging the uptake of hygiene and social distancing behaviours. Six studies demonstrated a relationship between greater levels of concern and compliance to recommended health behaviors (Hasan et al., 2018; Hussain et al., 2012; Mitchell et al., 2011; Seale et al., 2012; Serino et al., 2011; Van et al., 2010). Students with high levels of concern complied with behaviours such as avoiding crowded places (Serino et al., 2011), cancelling their travel plans (Serino et al., 2011), handwashing (Seale et al., 2012), avoiding people who appeared to be ill (Mitchell et al., 2011), and displaying appropriate cough etiquette (Seale et al., 2012). Furthermore, a study conducted in Italy found that $67 \%$ of the 'very worried' students adopted a number of health behaviors, which contrasts with only $18 \%$ of 'not worried' students who adopted at least one health behavior (Serino et al., 2011). Furthermore, 32\% of 'very worried' students from Italy reported that they would cancel their travel plans to the United States to avoid the risk of infection compared to only $5 \%$ of students who did not express worry (Serino et al., 2011). Consequently, 59\% of students reported not modifying their behavior to reduce their risk of infection (Serino et al., 2011). One study reported that risk perception was directly associated with overreaction to the behavior of oneself and others. However, risk perception was not correlated with compliance with self-quarantine (Yang and Cho, 2017). This study found that risk perception motivates self-protective behaviour rather than behaviors that protect others such as quarantining (Yang and Cho, 2017).

\section{Misconceptions}

Three studies identified a lack of clarity about the different clinical manifestations of seasonal influenza, swine flu and the common cold among university students (Akan et al., 2010; Park et al., 2010; Serino et al., 2011). For example, $13 \%$ of university students from Italy and $50.5 \%$ of those from Turkey indicated that swine influenza was less serious than seasonal influenza (Akan et al., 2010; Serino et al., 2011). A similar pattern was also noted in another study conducted in South Korea which reported that $56.1 \%$ of university students believed that H1N1 caused symptoms that resembled those of the common cold but were milder in nature (Park et al., 2010). 
Misconceptions regarding the transmission of disease were also prevalent. While $39 \%$ of medical and dental students thought that $\mathrm{H} 1 \mathrm{~N} 1$ can be contracted through contaminated food and water, $35.2 \%$ believed that eating cooked pork was a potential means of transmission (Hasan et al., 2018). The same study also reported that approximately a tenth of university students inaccurately identified sexual intercourse and mosquitoes as modes of transmission of the disease (Hasan et al., 2018). Similarly, $70.9 \%$ of students from Saudi Arabia were found to falsely believe that the consumption of raw or insufficiently cooked animal products, including dairy products and meat, was unsafe in the context of the MERS outbreak (Al-Mohaissen, 2016).

Students across various studies also demonstrated confusion regarding the origin of infection, classification of pandemics, and their support for conspiracy theories. For example, in one study, some university students from Iran suggested that EVD had been produced in the West to be tested in Africa (Holakouie-Naieni et al., 2015). Moreover, some university students in a study conducted in Pakistan incorrectly indicated that $\mathrm{H} 1 \mathrm{~N} 1$ was caused by a bacterium or that it was a heritable condition (Hasan et al., 2018). Students were also misled about the severity of disease with over half of those from a university in Turkey suggesting that H1N1 infection would result in death (Akan et al., 2010).

\section{Vaccination}

University students in three studies reported their willingness to vaccinate against $\mathrm{H} 1 \mathrm{~N} 1$ (Akan et al., 2010; Serino et al., 2011; Van et al., 2010). The proportion of students ranged from 7.2\% in Turkey (Akan et al., 2010) to $60 \%$ of students in Australia (Van et al., 2010). Despite the low willingness to vaccinate in some regions, $54 \%$ of university students believed that the vaccine could curb the spread of H1N1 (Akan et al., 2010). Students in two studies conducted in Turkey and Italy reported that the low willingness to vaccinate was attributed to a lack of trust in the vaccine, and uncertainty of its efficacy and potential side-effects (Akan et al., 2010; Serino et al., 2011). Furthermore, the low willingness to undergo vaccination may be due to the $59.6 \%$ of students who believed that insufficient information had been provided regarding the H1N1 vaccine (Akan et al., 2010). Furthermore, a study in Pakistan found that only $1.7 \%$ of medical students were vaccinated against H1N1 (Hasan et al., 2018). Two studies conducted in Australia and the United States reported variables that influenced seasonal flu vaccination (Van et al., 2010; Zottarelli et al., 2012). Van et al. (2010) reported that students who had received seasonal influenza vaccinations in the past were significantly more likely to accept the H1N1 vaccine compared to their nonvaccinated counterparts. Finally, a study conducted in the United States reported a positive correlation between frequent hand sanitizing and seasonal flu vaccination (Zottarelli et al., 2012).

\section{Discussion}

Summary of Findings

This systematic review examined the findings of 18 studies on the knowledge, awareness, risk perceptions and preventive behaviors of students in higher education institutions across 15 countries. The included studies cover three major pandemics and global outbreaks in the 21 st century: Ebola, 
Influenza A/H1N1, and MERS. In the following section, we analyze the findings from our systematic review and discuss potential interventions or strategies that can help to curb the spread of disease among students and increase compliance to appropriate health behaviors. We employ the InformationMotivation-Behavioral Skills (IMB) model to develop future policies that may help to reduce high-risk behavior among students.

\section{Risk Perception, Unrealistic Optimism, and Preventive Behavior}

Past literature has reported that risk perception contains an affective component which involves fear or worry about the health problem (Kiviniemi and Ellis 2013). The Protection Motivation Theory was developed to examine how individuals protect themselves against health threats (Rogers 1975). According to the Protection Motivation Theory, individuals who perceive higher levels of risk are more likely to adopt preventive behaviors (Prentice-Dunn \& Rogers, 1986). In this study, we found that the majority of students possessed low levels of concern in response to the pandemic which translated to lower adherence to preventive behaviors (Hasan 2018; Holakouie-Naienia 2015; Hussain 2012; Van 2010). Low levels of concern and worry were attributed to students' perception of their "invincible and immune" bodies, which were deemed to be incapable of contracting viruses (Seale 2012). Previous research has found that students are more likely to make inaccurate or unstable judgments about selfrisk and behavioral control compared to the general population (Notani 1998). For example, a study that explored possible roles for optimistic bias in the Integrative Model of Behavioral Prediction on a university campus during the 2009 H1N1 pandemic supported this idea (Kim and Niederdeppe 2012). This study found that unrealistic optimists (i.e., individuals who believed that their risk is lower or equal to others) had significantly lower intentions to execute hand hygiene practices compared with others who believed that their risk was higher than other groups (Kim and Niederdeppe 2012). Another study found that students were less likely to follow precautionary measures when they believed that information from the media related to the pandemic was not relevant to them (Lee 2016). A lack of concern related to pandemic influenza may decrease risk perception and hence decrease adherence to preventive behaviors.

\section{Minimizing High-Risk Behavior}

A possible model that can be used as a framework to minimize pandemic high-risk behaviors and promote preventive behaviors among university students is the Information-Motivation-Behavioral skills (IMB) model (Fisher and Fisher, 1992). The IMB model conceptualizes that three fundamental determinants - health-related information, motivation to avoid the disease and health behavioral skills are necessary to promote and reinforce prevention behavior (Fisher and Fisher, 1992). This model has been statistically validated and applicable in young adults in the United States (Bazargan et al., 2010; Brown et al., 2016; Glasford 2008), and can serve as a framework to develop health-based intervention strategies for students in higher education institutions.

The first construct (health-related information) of the model claims that information levels about prevention behaviors is a vital but not sufficient condition for behavior change, especially when the 
behavior is complex in nature (Robinson 2017). To understand the IMB framework, it is important to define the terms "incremental theorists" and "entity theorists." Incremental theorists believe that individuals are malleable and can evolve through action whereas entity theorists believe that people are fixated and cannot change. Given the distinction between incremental and entity theorists an intervention that promotes preventive behaviors during pandemics may include the following: claims that certain hygiene behaviors and physical distancing guidelines prevent viral transmission, myths pertaining to vaccination, and the implicit theory that "incremental-theorists will perceive greater self-efficacy compared to entity theorists" (Zhang et al., 2020). These three pieces of information are based on the original IMB model which was found to produce a powerful effect in the adoption of HIV preventive behaviors in truck drivers in India (Cornman 2007). Similarly, a study conducted in Jordan during the COVID-19 pandemic found that high levels of symptom and vaccination knowledge was due to the effectiveness and reach of various awareness campaigns (Alzoubi 2020). A number of studies in this review indicated that higher levels of knowledge about modes of transmission, symptoms, and preventive methods led to a greater uptake of hygiene behaviors and compliance with self-quarantine guidelines (Yang 2017; Zottarelli 2012). However, one study in this review found that despite public health and university health education campaigns, approximately $25 \%$ of students were unaware of the virus (Wilson 2010). This finding reinforces the importance of utilizing personalized health education strategies that are consistent with the mechanisms that students use to gain health information (Wilson 2010). Although social media was a prominent information source in a number of included studies, it often lacked the depth and organization to reliably implement an educational curriculum. Hence, personalized education based on clinical practice guidelines, reminders and social media support may effectively inform students about prevention behaviors. This may include the development of social media apps that can connect students to government COVID-tracking sites and other resources. In addition, teachers and administrators of education can also play a more active role in information dissemination.

Encouragement of verbal communication and discussion-based learning can help to facilitate a better understanding of the disease and aid students in becoming aware of the purpose behind recommended health measures. Moreover, having more of these discussions can create an environment where students can clarify any misconceptions and be led to use the right information sources.

The second construct (motivation to avoid the disease) of the model pertains to personal motivation (i.e., perceptions of intervention efficacy and health behavior attitudes) and social motivation (i.e., perceived social support or social norms surrounding behavior) (Fisher and Fisher 1992). The influence of personal motivation on behavior change was demonstrated in a study conducted in the United States on the general population during the COVID-19 pandemic, which found that adherence to non-pharmaceutical interventions (NPI) was associated with both outcome expectations (i.e., NPIs are effective) and process expectations (i.e., NPIs are inconvenient) (Kantor and Kantor 2020). Another study conducted in Jordan during COVID-19 found that only $19.3 \%$ of medical students believed face masks to be preventive, which correlated with $61 \%$ of students who did not wear face masks during the pandemic (Khasawneh 2020). Low process expectations were also evident within this review where students were most resistant to 
comply to social avoidance interventions as they were deemed to be impractical due to the regularity of human interaction in society (Seale 2012). Furthermore, international university students considered maintaining distance from infected acquaintances or relatives to be impolite (Seale 2012). At the same time, due to low process expectations, the use of masks were considered uncomfortable, inconvenient, and in some cases, unnecessary (Seale 2012). Being aware of reasons for NPI refusal among students may enable policymakers to tailor their messaging accordingly and induce behavioral change. Hence, understanding the relationship between intervention outcome beliefs and adherence to preventive behavior is essential as knowledge gaps of NPI efficacy can be addressed through outreach efforts. One study in this review claimed that perception of media influence on self is the most important indicator of consequent attitudinal and behavioral changes (Lee 2016). In this sense, regardless of the severity of a news story, unless people perceived the story as personally relevant to them and were confident in their ability to perform the suggested behaviors, the sensationalization of a news story may have no meaningful difference in behavior change.

The third construct (health behavioral skills) in the IMB model pertains to skills necessary for the execution of health behaviors. A study by Fisher et al. reported that the facilitation of behavioral change is induced by an increase in perceived self-efficacy and enhancement of an individual's objective skills. A study conducted by Zhang et al. 2020 claimed that an individual's implicit theory orientation serves as an important determinant of their extent and timing of their prevention behaviors. For example, individuals who endorse an incremental theory believe in their general abilities to evoke change more so than do entity-theorists, hence these individuals should exhibit higher levels of perceived self-efficacy (Zhang et al., 2020). This correlation is suggested but not directly tested in the health behavior literature.

\section{Implications of this Research}

In the face of public health crises, the IMB model can serve as a framework for the construction of effective interventions. Policymakers and government officials should pay attention to publicity and guidance, strengthen positive interactions with the public and disclose relevant information in a timely manner to gain trust and to maintain the positive public perception of the interventions. Health education campaigns should target behavioral skills, strive to mitigate ineffective prevention information and raise awareness about the disease in order relieve stress and anxiety among university students. The findings of this review may be used to construct better communication policies in order to manage future pandemics and outbreaks on university campuses. Taking into consideration the negative relationship between unrealistic optimism and intention to adhere to preventive behaviors, it is also vital to inform students of risk factors that lead to infection, so students are aware of the relationship between perceived and actual risk.

\section{Strengths and Limitations}

This systematic review has many strengths. This review explored a broad spectrum of university student campuses from various countries which allowed for a diverse extraction of behavior analysis from each 
group. This may lead to a greater degree of transferability of our findings. Our findings may be applicable when discussing, constructing and enforcing higher education health policies that are reflective of the behavior of students. Furthermore, this review may also be beneficial in gauging the knowledge levels of students. It may thus have implications for implementation of campaigns specifically tailored to this group, allowing for a more efficient communication of pandemic-related information.

Despite the many strengths of this systematic review, we identify a number of limitations. This review was not able to examine all the possible pandemics, epidemics and outbreaks that occurred during the 21 st century. Three major pandemics were selected based on their global impact, transmission method, and disease epidemiology. Moreover, pandemics and epidemics prior to the 21st century were not analyzed, which may have provided important insights into how knowledge, awareness, risk perceptions and health behaviors have changed with time.

\section{Declarations}

\section{Conflicts of Interest}

Umair Majid receives financial support from the Canadian Institutes of Health Research and the Government of Ontario, Canada. Neither party was involved in the design and conduct of this research.

\section{References}

1. Akan H, Gurol Y, Izbirak G, Ozdatlı S, Yilmaz G, Vitrinel A and Hayran O (2010) Knowledge and attitudes of university students toward pandemic influenza: A cross-sectional study from Turkey. BMC Public Health 10(1): 413.

2. Alhamid, AM (2020) Knowledge and behaviors towards COVID-19 among University of Aleppo students: an online cross-sectional survey. medRxiv.

3. Al-Mohaissen M (2017) Awareness among a Saudi Arabian university community of Middle East respiratory syndrome coronavirus following an outbreak. Eastern Mediterranean Health Journal 23(5): 351-360.

4. Alzoubi H, Alnawaiseh N, Al-Mnayyis A, Lubad MA, Aqel A and Al-Shagahin H (2020) COVID-19knowledge, attitude and practice among medical and non-medical University Students in Jordan. $J$ Pure Appl Microbiol 14(1):17-24.

5. Aronson J (1995) A pragmatic view of thematic analysis. The Qualitative Report 2(1): 1-3

6. Bazargan M, Stein JA Bazargan-Hejazi S and Hindman DW (2010) Using the Information-Motivation Behavioral Model to Predict Sexual Behavior Among Underserved Minority Youth. Journal of School Health 80(6): 287-

7. Boeije $\mathrm{H}$ (2002) A purposeful approach to the constant comparative method in the analysis of qualitative interviews. Quality and Quantity 36(4): 391- 
8. Brown W, Carballo-Diéguez A, John RM and Schnall R (2016) Information, motivation, and behavioral skills of high-risk young adults to use the HIV self-test. AIDS and Behavior 20(9): 2000-

9. Cornman DH, Schmiege SJ, Bryan A, Benziger TJ and Fisher JD (2007) An information-motivationbehavioral skills (IMB) model-based HIV prevention intervention for truck drivers in India. Social Science \& Medicine 64(8): 1572-

10. Fisher JD and Fisher WA (1992) Changing AIDS-risk behavior. Psychological Bulletin 111(3): 455.

11. Glasford DE (2008) Predicting voting behavior of young adults: The importance of information, motivation, and behavioral skills. Journal of Applied Social Psychology 38(11): 2648-

12. Hasan F, Khan MO and Ali M (2018) Swine flu: Knowledge, attitude, and practices survey of medical and dental students of Karachi. Cureus 10(1): e2048.

13. Holakouie-naieni O, Ahmadvand A, Raza O, Assan A, Elduma A, Jammeh A, Kamali ASMA, Kareem AA, Muhammad FM, Sa-Bahat H, Abdullahi KO, Saeed RA and Saeed SN (2015) Assessing the knowledge, attitudes, and practices of students regarding Ebola virus disease outbreak. Iranian Journal of Public Health 44(12): 1670-1676.

14. Hussain ZA, Hussain SA and Hussain FA (2012) Medical students' knowledge, perceptions, and behavioral intentions towards the H1N1 influenza, swine flu, in Pakistan: A brief report. American Journal of Infection Control 40(3): e11- e13.

15. Kantor BN and Kantor J (2020) Nonpharmaceutical interventions for pandemic COVID-19: A crosssectional investigation of US general public beliefs, attitudes, and actions. MedRxiv.

16. Khasawneh Al, Humeidan AA, Alsulaiman JW, Bloukh S, Ramadan M, Al-Shatanawi TN, Awad HH, Hijazi WY, Al-Kammash KR, Obeidat N and Saleh T (2020) Medical students and COVID-19: Knowledge, attitudes, and precautionary measures. A descriptive study from Jordan. Frontiers in Public Health 8: 253

17. Kim HK and Niederdeppe $J$ (2013) Exploring optimistic bias and the integrative model of behavioral prediction in the context of a campus influenza outbreak. Journal of Health Communication 18(2): 206-222.

18. Kiviniemi MT and Ellis EM (2014) Worry about skin cancer mediates the relation of perceived cancer risk and sunscreen use. Journal of Behavioral Medicine 37(6):1069-1074.

19. Lee H and Park S (2016) Third-person effect and pandemic flu: The role of severity, self-efficacy method mentions, and message source. Journal of Health Communication 21(12): 1244-1250.

20. Lee SW, Schwarz N, Taubman D and Hou M (2010) Sneezing in times of a flu pandemic: Public sneezing increases perception of unrelated risks and shifts preferences for federal spending. Psychological Science 21(3): 375-377.

21. Majid U, Bakshi S, Truong J and Wasim A (2020a) Sustaining Social Distancing and Mass Quarantine in the COVID-19 Era: Lessons Learned. Healthcare Quarterly 23(2): 21-23.

22. Majid U, Wasim A, Bakshi S and Truong J (2020b) Knowledge, (mis-) conceptions, risk perception, and behavior change during pandemics: A scoping review of 149 studies. Public Understanding of Science 29(8): 777-799. 
23. Mitchell T, Dee DL, Phares CR, Lipman HB, Gould LH, Kutty P, Desai M, Guh A, luliano AD, Silverman P, Siebold J, Armstrong GL, Swerdlow DL, Massoudi MS, and Fishbein DB (2011) Non-pharmaceutical interventions during an outbreak of 2009 pandemic influenza A (H1N1) virus infection at a large public university, April-May 2009. Clinical Infectious Diseases 52(S1): S138-S145.

24. Mondragon NI, de Montes LG and Valencia J (2017) Understanding an Ebola outbreak: Social representations of emerging infectious diseases. Journal of Health Psychology 22(7): 951-960.

25. Notani AS (1998) Moderators of perceived behavioral control's predictiveness in the theory of planned behavior: A meta-analysis. Journal of Consumer Psychology 7(3): 247-271.

26. Park J, Cheong H, Son D, Kim S and Ha C (2010) Perceptions and behaviors related to hand hygiene for the prevention of $\mathrm{H} 1 \mathrm{~N} 1$ influenza transmission among Korean university students during the peak pandemic period. BMC Infectious Diseases 10(1): 222.

27. Prentice-Dunn S and Rogers RW (1986) Protection motivation theory and preventive health: Beyond the health belief model. Health Education Research 1(3): 153-161.

28. Robinson WT (2017) Adaptation of the Information-Motivation-Behavioral Skills Model to Needle Sharing Behaviors and Hepatitis C Risk: A Structural Equation Model. Sage Open 7(1): 2158244016666126.

29. Rogers RW (1975) A protection motivation theory of fear appeals and attitude change1. The Journal of Psychology 91(1): 93-114.

30. Seale H, Mak JP, Razee H and Maclntyre CR (2012) Examining the knowledge, attitudes and practices of domestic and international university students towards seasonal and pandemic influenza. $B M C$ Public Health 12(1): 307.

31. Seale H, McLaws M, Van D, Crimmins J, and Maclntyre C (2011) University communication strategies during a pandemic-were the messages received? Journal of Public Health Management and Practice 17(1): E29-E32.

32. Serino L, Meleleo C, Maurici M, Bagnato B, Sorbara D, Zaratti L and Franco E (2011) Knowledge and worry as basis for different behaviors among university students: the case of Pandemic Flu H1N1v. Journal of Preventive Medicine and Hygiene 52(3): 144-147.

33. Van D, McLaws M, Crimmins J, Maclntyre CR and Seale H (2010) University life and pandemic influenza: Attitudes and intended behaviour of staff and students towards pandemic (H1N1) 2009. BMC Public Health 10(1): 130.

34. Von Elm E, Altman DG, Egger M, Pocock SJ, Gøtzsche PC and Vandenbroucke JP (2007) The Strengthening the Reporting of Observational Studies in Epidemiology (STROBE) statement: Guidelines for reporting observational studies. Annals of Internal Medicine 147(8): 573-577.

35. Wadood MA, Mamun ASMA, Rafi MA, kamrul Islam M, Mohd S, Lee LL and Hossain MG (2020) Knowledge, attitude, practice and perception regarding COVID-19 among students in Bangladesh: Survey in Rajshahi University. medRxiv.

36. Williams L, Regagliolo A and Rasmussen S (2012) Predicting psychological responses to Influenza A, H1N1 ("Swine flu"): The role of illness perceptions. Psychology, Health \& Medicine 17(4): 383-391. 
37. Wilson SL and Huttlinger K (2010) Pandemic flu knowledge among dormitory housed university students: A need for informal social support and social networking strategies. Rural and Remote Health 10(4): 1526.

38. Yang $S$ and Cho S (2017) Middle East respiratory syndrome risk perception among students at a university in South Korea, 2015. American Journal of Infection Control 45(6): e53-e60.

39. Zhang Y, Mathur P and Block L (2020) Personality Matters during a Pandemic: Implicit Theory Beliefs Influence Preparedness and Prevention Behaviors. Journal of the Association for Consumer Research

40. Zottarellia LK, Sunil TS, Flott P and Karbhari S (2012) College student adoption of nonpharmaceutical interventions during the $2009 \mathrm{H} 1 \mathrm{~N} 1$ influenza pandemic: A study of two Texas universities in fall 2009. Preventive Medicine 55(5): 497-499.

\section{Supplementary Files}

This is a list of supplementary files associated with this preprint. Click to download.

- SupplementalFile.docx 